\title{
ON THE ORIGIN OF PLANETARY NEBULAE
}

\author{
G. O. ABELL ${ }^{*}$ AND P. GOLDREICH \\ Department of Astronomy \\ University of California, Los Angeles
}

Received March 5, 1966

There has been much interest of late in the evolution of planetary nebulae; recent papers on the subject include those of O'Dell (1962, 1963), Harmon and Seaton (1964), and Abell (1966). The detailed tracks of evolution are not yet clear, but the evidence strongly suggests that the central stars evolve directly into white dwarfs.

There has been less quantitative discussion of the origin of planetary nebulae. Shklovskiı (1956) suggested that planetary nebulae are formed from the outer layers of some (but not all) red giants, and he questioned whether the $\mathrm{T}$ Tauri stars, or such combination variables (or "symbiotic" stars) as $\mathrm{Z}$ Andromedae or $\mathrm{V}$ Sagittae might not be intermediate stages in the planetary nebulae formation. His arguments that red giants are the immediate precursors of planetary nebulae are based largely on following the evolution of a typical nebular shell backward in time and recognizing that at an early epoch its physical structure must resemble the very tenuous envelope of a greatly distended red giant. More recently, Deeming (1965) has similarly tried to trace the evolution of a planetary nebula backward, but he does not seem to specifically conclude that the nebulae are actually ejected from red giants. (Moreover, Deeming's model for the early appearance of a planetary nebula cannot be correct, for the forbidden radiation does not disappear at a critical gas density as he has assumed.) A possible relation between planetary nebulae and red giants, and in particular the "symbiotic" stars, has also been hinted at by Menzel (1946), Aller (1954), and Merrill (1958), and possibly others.

In our opinion there are some rather compelling arguments that most, or more probably all, population II red giants ultimately become planetary nebulae and further that the nebulae are formed directly from giant stars. To our knowledge, all of these arguments

* At the Institut für Astrophysik, Max-Planck-Institut für Physik und Astrophysik, Munich, Germany, 1965-66. 
have not been stated in the literature or at least have not been given in one place. In any case, it is our experience that many investigators working in the field are not familiar with the arguments, and it is the purpose of this communication to summarize them.

a) Most, if not all, planetary nebulae represent stages of evolution of stars of about $1.2 \mathfrak{M}_{\odot}$ after they have left the main sequence. The galactic distribution of planetary nebulae matches that of old stars (population II) such as those found in the nucleus, disk, and corona of the Galaxy and not that of the young stars found in or near the spiral arms (Minkowski and Abell 1963). It cannot be ruled out that some nebulae evolve from population I stars, but at least the vast majority must originate from older stars whose masses must be near $1.2 M_{\odot}$. This point has been well discussed by O'Dell (1963).

b) Most, if not all, stars of $1.2 M_{\odot}$, which are now evolving or have recently evolved off the main sequence, must ultimately pass through the planetary nebula stage. To verify this point, we need only compare the current rate at which stars in the requisite mass range are now leaving the main sequence to the rate of formation of planetary nebulae.

The present rate at which stars are leaving the main sequence is easily found from data given by Schmidt (1963), with main sequence lifetimes taken from Limber (1960). According to Schmidt, the present rate of formation of stars of mass near $1.3 \% \%_{\odot}$ is about $10^{-10}$ stars per magnitude interval per year in a column of one square parsec cross-section perpendicular to the galactic plane at the position of the sun. If stellar formation and evolution in the Galaxy has reached a steady state, this should also be the rate at which stars are leaving the main sequence. From Schmidt's data, we find that stars in the mass range 1.2 to $1.4 M_{\odot}$ (comprising a range of about 0.7 magnitude) should be leaving the main sequence

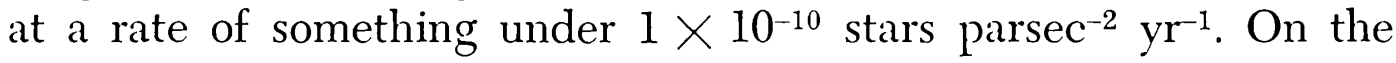
other hand, the rate of star formation was probably higher when stars of this mass were first formed (about $5 \times 10^{9}$ years ago). As an extreme alternative, suppose that all stars were formed at once, and that just now those of mass $1.2 \Re_{\odot}$ (potential parents of planetary nebulae) are leaving the main sequence. Then the rate of evolution from the main sequence should be $\mathrm{d} N / \mathrm{d} t=\phi\left(M_{\mathrm{r}}\right) \mathrm{d} M_{\mathrm{V}} /$ 
$\mathrm{d} t$, where $\phi\left(M_{V}\right)$ is the present luminosity function. We estimate $\phi\left(M_{V}\right)$ and $\mathrm{d} M_{V} / \mathrm{d} t$ from Schmidt's Table 1 , and find $\mathrm{d} N / \mathrm{d} t \approx$ $2 \times 10^{-10}$ stars parsec ${ }^{-2} \mathrm{yr}^{-1}$. Thus the present rate of supply of stars that can become planetary nebulae (in our part of the Galaxy) probably lies in the range $1 \times 10^{-10}$ and $2 \times 10^{-10}$ stars parsec $^{-2} \mathrm{yr}^{-1}$. Adopting $75 \mathfrak{M}_{\odot}$ per square parsec as the total surface density of the Galaxy at the sun's position (Schmidt 1963) and $1.1 \times 10^{11} \mathfrak{M}_{\odot}$ for the mass of the Galaxy (Allen 1963), we find that in the entire Galaxy the rate of such stars leaving the main sequence should be less than 0.3 stars per year. Even if we assume that the formation and evolution of all stars in a steady state in all parts of the Galaxy - an assumption which must certainly greatly exaggerate the rate of production of red giants - we find from Schmidt's data that the total rate at which stars of all mass greater than $1.2 \mathfrak{M}_{\odot}$ leave the main sequence in the entire Galaxy is not over about 0.7 stars per year.

The total number of planetary nebulae in the Galaxy is more difficult to determine. O'Dell (1963) estimates that there are $5 \times 10^{4}$ nebulae with radii $R \leq 0.7$ parsec. Shklovskii (1956) gives a similar estimate. Typical nebulae expand about their parent stars at velocities of about $30 \mathrm{~km} / \mathrm{sec}$ (Wilson 1950). The lifetime of a nebula (to reach a radius of 0.7 parsec) is therefore typically about $2 \times 10^{4}$ years. If O'Dell's estimate is correct, planetary nebulae are now forming in the Galaxy at the rate of 2 or 3 per year. Since nebulae cannot form faster than the stars from which they are ejected become available, there are very likely fewer nebulae than $5 \times 10^{4}$. There can hardly be less than about $5 \times 10^{3}$, however, since nearly a thousand planetaries are now known. Thus it seems inescapable that most or all stars of mass near $1.2 \mathfrak{M}_{\odot}$ or slightly greater - those stars that are now leaving or have recently left the main sequence throughout the disk population of the Galaxy - will become planetary nebulae.

c) The observed typical expansion velocity cannot be a significant underestimate due to observational selection. If some planetary nebula shells were ejected very rapidly, so that their expansion speeds were in excess of $100 \mathrm{~km} / \mathrm{sec}$, they would have short lifetimes. The question might arise, therefore, whether we preferentially discover only those nebulae of relatively low expansion 
velocity. However, there cannot be an appreciable fraction of nebulae expanding at speeds in excess of $100 \mathrm{~km} / \mathrm{sec}$, for at least two reasons: (1) The mean lifetimes for the nebulae would then be substantially less than $2 \times 10^{4}$ years, and their formation rate would have to be even greater than the estimate given above, which already seems to exceed the rate at which precursors of planetary nebulae become available in the Galaxy. (2) If, say, all expansion speeds between 10 and $1000 \mathrm{~km} / \mathrm{sec}$ were equally likely, since the probability of observing a nebula is proportional to its lifetime, the probability that a randomly observed nebula would have a velocity greater than $100 \mathrm{~km} / \mathrm{sec}$ would be $\log 10 / \log 100=$ $1 / 2$ - that is, approximately half of the measured expansion speeds should exceed $100 \mathrm{~km} / \mathrm{sec}$. Actually, of 25 velocities listed by Wilson (1950), only one is over $100 \mathrm{~km} / \mathrm{sec}$.

d) The mechanism of ejection of a nebular shell must arise from internal conditions in the star producing a more or less ballistic push, rather than a continual acceleration after the nebula has left the star. Radiation pressure and a continuous ejection of a "stellar wind" of corpuscular radiation from the central star probably influence the internal dynamics in a nebular shell, at least at some stages of its evolution (Mathews 1966), but neither of these can be the primary ejection mechanism. Even if the gas remained optically thick to such electromagnetic and/or corpuscular radiation, the amount of momentum required to be carried by that radiation would be enormous. Typical nebular shells have masses of $0.2 \mathfrak{M}_{\odot}$ (O’Dell 1962, Osterbrock 1964). Just to support the weight of such a shell at the surface of a star of one solar mass and radius $R$ with the momentum carried by radiation, the luminosity of the star would have to be

$$
L=8.3 \times 10^{13} / R^{2}
$$

where $L$ and $R$ are in solar units. Luminosities and radii compatible with equation (1) are outside the range of those possessed by known stars. A shell might be produced by successive ejections of smaller mass, but in any case the minimum momentum transfer required to remove a unit mass from a distance $r$ from a gravitating body to infinity is easily shown to be the velocity of escape from 
that body at the distance $r$. Thus the total momentum needed to blow out a shell of mass $0.2 \mathfrak{M}_{\odot}$ is at least $0.2 \mathfrak{M}_{\odot}\left(2 G \mathfrak{M}_{\odot} / R\right)^{1 / 2}$, which, for $R$ in solar units, is $2.5 \times 10^{40} / R^{1 / 2} \mathrm{gm} \mathrm{cm} \mathrm{sec}^{-1}$. To supply this momentum in the form of radiation pressure would require a luminous output equivalent to $6 \times 10^{5} L_{\odot}$ spread over $10^{4}$ years if the mass were ejected from a star of $1 R_{\odot}$, and $2 \times 10^{4} L_{\odot}$ over the same time even if all of the mass were ejected from a giant star of $10^{3} R_{\ominus}$; such stars fall far outside the range of properties of observed planetary nebulae nuclei. Indeed, just to accelerate a mass of $4 \times$ $10^{32} \mathrm{gm}$ from rest to a speed of $30 \mathrm{~km} / \mathrm{sec}$ in $10^{4}$ years, without any gravitational drag whatsoever, would require a luminous output over that time of $3 \times 10^{4} L_{\odot}$.

Moreover, even if radiation pressure were to be effective in accelerating a nebular shell, that shell would have to be optically thick to the radiation. The repulsive force on the nebula would thus remain approximately constant, while the force of gravity on the expanding shell would drop off as $1 / r^{2}$. Ultimately, the nebular shells would be accelerated to very high velocities unless they either became transparent to the driving radiation or that radiation diminished in intensity at just the right moment to leave a nebula with essentially the velocity of escape from its star. It is difficult to imagine that such a coincidence could occur in every planetary nebula.

Whereas there is little observational basis for estimates of the momentum that can be carried by stellar winds from planetary nuclei, it appears almost out of the question that corpuscular radiation could blow $0.2 M_{\odot}$ away from anything but a giant star by gradual acceleration after the star and shell have separated. Even if all of the energy of the ejected mass (in the corpuscular wind) could be converted into mechanical thrust on the planetary shell, a simple analysis of the energy requirements shows that at the very minimum, a corpuscular flux equivalent to $10^{3} L_{\odot}$ would have to work on the shell to eject it in $10^{4}$ years from a star of $1 R_{\odot}$, and then only if the stellar wind were ejected with a speed of at least several times the escape velocity. These arguments do not rule out that corpuscular radiation could play an important role in the ejection of a substantial mass from a star of very large radius, but this 
possibility, of course, is not inconsistent with our general conclusions.

e) Stellar rotation is not important in the ejection of planetary nebulae. Deeming (1965) has proposed a model in which stellar rotation plays a role in the ejection of the nebula. He points out that the rotational energy in a rapidly rotating star (say, $100 \mathrm{~km} / \mathrm{sec}$ at the equator) is of the order of $10^{47} \mathrm{ergs}$, and that this is near the energy required to remove a nebular shell to infinity. If a major part of the stellar mass contracts to a small dense core while the outer stellar envelope grows (as in a star evolving to a red giant), the core acquires a high rotation rate compared to the envelope. Now if rotational energy can be transferred from the core to the envelope (Deeming suggests a deep subsurface convection zone and magnetic fields), part of the envelope, according to Deeming's model, gains enough rotational energy to be ejected. Deeming feels that if just enough rotational energy is given to the shell to get the ejection started, the nebular matter can be further accelerated to the escape velocity by Lyman- $\alpha$ absorption.

We have seen, however, that no form of radiation pressure can be important in ejecting a planetary nebula shell. There are, moreover, several reasons why rotation cannot be important either. First, the transfer of energy from the core to the envelope would have to be sudden, for a gradual increase in rotation of the envelope would at best merely expand and flatten it, not eject it. Moreover, we would expect the material to come off in a ring from the equatorial regions of the star, not in a shell, as is observed. Nor could material in a ring diffuse into a shell distribution after ejection, for the expansion velocities of the planetary nebulae are typically at least 2 or 3 times the thermal velocities in a gas at $10^{4}{ }^{\circ} \mathrm{K}$.

The main argument against the rotation model for nebular ejection is that the star can never have enough angular momentum. The minimum momentum, $p$, needed to remove the nebular material to infinity is, as we have seen, $0.2 \mathfrak{M}_{\odot}\left(2 G \mathfrak{M}_{\odot} / R\right)^{1 / 2}$, where $R$ is a representative radius in the matter to be ejected. For circular motion, the angular momentum required is thus $p R=0.2 \mathfrak{M}_{\odot}\left(2 G \mathfrak{M}_{\odot}\right)^{1 / 2} R^{1 / 2}$. Now for an initially rigidly-rotating star, the angular momentum is $I v / R_{*}$, where $I$ is the moment of inertia, $v$ is the equatorial speed at the surface of the star, and $R_{*}$ 
is the radius of the star before evolutionary effects produce any differential rotation. Thus a necessary (but not sufficient) condition for ejection of the shell is

$$
I v / R_{*} \supseteq 0.2 \mathfrak{M}_{\odot}\left(2 G \mathfrak{M}_{\odot}\right)^{1 / 2} R^{1 / 2}
$$

It is instructive to express $v$ in terms of that circular velocity at the stellar surface at which the star is barely rotationally stable. If the star before nebular ejection has a mass of $1.2 \mathfrak{M}_{\odot}$,

$$
v_{\mathrm{cir}}=\left(6 G \mathfrak{M}_{\odot} / 5 R_{*}\right)^{1 / 2}
$$

so that (from equation (2))

$$
\frac{v}{v_{\mathrm{cir}}} \geq \frac{0.2 \mathfrak{M} \mathcal{R}_{\odot} R^{1 / 2}}{I}\left(5 / 3 R_{*}^{3}\right)^{1 / 2}
$$

Inspection of published models of stars, both homogenous and inhomogeneous, in the mass range 0.6 to $2.5 \mathfrak{M}_{\odot}$ (Schwarzschild 1958 ), shows that the central concentration of density in actual stars is very much underestimated by a density law of the form

$$
\rho(r)=k\left(1-r / R_{*}\right)^{2} .
$$

Thus the moment of inertia computed from equation (5), which is $8 \mathfrak{M}_{\odot} R_{*}^{2} / 35$, is an overestimate of $I$. The inequality, equation (4), thus becomes

$$
\frac{v}{v_{\mathrm{cir}}} \geq 1.3\left(R / R_{*}\right)^{1 / 2}
$$

Since the envelope would grow in a star whose core contracted, giving rise to the needed differential rotation $R / R_{*}>1$, we find that the initial star would have to be rotationally unstable. This argument does not, of course, rule out the possibility of a large star contracting and shedding mass (in the form of an equatorial disk). The equatorial velocity of such a star, however, never exceeds the circular velocity; thus it cannot, according to equation (6), send $0.2 \mathfrak{M}_{\odot}$ into a parabolic orbit. 
f) The ejection of a planetary nebula shell almost certainly occurs from a star of extremely large radius. The velocity of escape at a distance of 0.1 parsec from a star of $1 M_{\odot}$ is only about $0.3 \mathrm{~km} /$ sec, which is negligible compared to the observed expansion speeds of planetary nebulae. Thus the nebular shells now have virtually the same kinetic energy, $E_{\infty}$, that they will have when they have expanded to infinity. (We ignore the possibility of drag effects of interstellar matter because the galactic distribution of planetary nebulae places them in locations where such matter is not expected to perturb appreciably their expansion.) If $E_{0}$ is the kinetic energy of the shell at ejection,

$$
E_{0}=E_{\mathrm{cs}}+E_{x}
$$

where $E_{\text {ss }}$ is the escape energy from the star at the time of ejection. Assuming $1.0 \% \eta_{\odot}$ and $0.2 M_{\odot}$ for the star and shell, respectively, we find that $E_{\mathrm{es}}=7.7 \times 10^{47} / R$ ergs, where $R$, the radius of the star at ejection, is expressed in units of the solar radius, and the ratio $E_{\mathrm{cs}} / E_{\infty}$ is about $400 / R$. If the central stars with their present observed sizes $(R<0.01$ to 1$)$ had ejected the nebular shells, $E_{\text {cs }}$ would have to exceed $E_{x}$ by factors of the order of nearly $10^{3}$ to $10^{5}$. The large disparities between $E_{\mathrm{es}}$ and $E_{\infty}$ would appear to rule out sudden ejection from small or solar-sized stars as the mechanism giving rise to the planetary nebulae, for it is difficult to understand how a star could throw off a nebula with the escape energy to within one part in several hundred or, in extreme cases, to one part in one hundred thousand. Shklovskii (1956) made the point that the ejection velocity of the nebula would have to be the escape velocity of the star; actually this is not true if the star is so very large that $E_{\mathrm{es}}$ and $E_{x}$ are comparable. We would, in fact, expect a spread in the energies of the ejecting mechanisms. For the dispersion in ejection energies to be comparable to a typical ejection energy itself, the stars would have to be as large as $400 R_{\odot}$.

We are therefore virtually forced to the conclusion that planetary nebulae are ejected more or less suddenly, although apparently not catastrophically, from stars of extremely large radii. Shklovskii hypothesizes that red giants simply expand until their envelopes "sluff off" at essentially zero velocity, becoming the nebular shells, while the stellar cores quickly evolve, readjusting themselves into 
the stars observed as the planetary nuclei. Shklovskil calls attention to the fact that observed expansion speeds (which he quotes to be of the order of $10 \mathrm{~km} / \mathrm{sec}$ ) do not greatly exceed the thermal velocities in a gas at $10^{4}{ }^{\circ} \mathrm{K}$. On the other hand, to obtain the observed hyperbolic velocities, which range up to $100 \mathrm{~km} / \mathrm{sec}$, a somewhat less leisurely event than that envisaged by Shklovskii might be required.

g) The parent stars of planetary nebulae are most probably red giants. If a star of 200 to $1000 R_{\odot}$ had a spectral class earlier than that of the sun, its absolute magnitude would have to be brighter than -6 to -10 . It is unlikely that such luminous stars with a population II or "disk" distribution in the Galaxy would escape detection unless their lifetimes are very short indeed; in fact novae (which do not give rise to planetaries) of lesser luminosity are commonly discovered. We must conclude that the most probable precursors of planetary nebulae are red giants.

\section{Conclusions}

Planetary nebulae must be ejected from extreme giant stars, probably red giants. Although Shklovskiı reached this same conclusion a decade ago, it seems to us that his arguments are less binding. Moreover, we conclude that all, or nearly all, stars of $1.2 \mathfrak{M}_{\odot}$ must eventually become planetary nebulae. The central stars of some planetary nebulae have spectra which according to Greenstein and Minkowski (1964), are not incompatible with the idea that they are relics of the interiors of red giants. The possibility that combination variables like $\mathrm{Z}$ Andromedae, in which bright lines appear to arise from a nebulosity in the vicinity of an M star, are early stages of the planetary nebula phenomenon has already been mentioned.

The ultimate fate of planetary nebulae nuclei appears to be white dwarfs. We are not aware that it has yet been shown that the very small nuclei observed for some planetaries are electron degenerate, but in any case it seems unlikely that these stars will re-grow envelopes and expand again. If planetary nebulae come from red giants and their nuclei evolve to white dwarfs, we are left with the problem of explaining the horizontal branch stars. Our estimate of the formation rate of planetary nebulae compared to that of red 
giants is not so precise that we can rule out the possibility that two paths of evolution are open to red giants, one leading to planetary nebulae and the other to a sequence of phases involving horizontal branch and RR Lyrae stars, but we can rule out the planetary phenomenon as a rare occurrence, accounting for only a small fraction of the red giants. Moreover, it would be strange that stars which, as far as is determined, have identical masses, chemical compositions, and other characteristics should not follow similar evolutionary patterns. We suggest that the possibility should be taken seriously that stars evolve through a giant phase at least twice - once immediately after leaving the main sequence, and again in a post-horizontal-branch phase, prior to becoming planetary nebulae.

We wish to thank Drs. L. H. Aller, D. M. Popper, E. K. Upton, J. Hazelhurst, and H. Schmidt for their helpful discussions with us concerning this interesting problem. One of us (P. G.) acknowledges support from NASA 216-62.

\section{REFERENCES}

Abell, G. O. 1966, Ap. J. 143, 259.

Allen, C. W. 1963, Astrophysical Quantities (London: Athlone Press), p. 267. Aller, L. H. 1954, Pub. Dominion Astrophysical Obs. 9, 321.

Deeming, T. J. 1965, Pub. A. S. P. 77, 443.

Greenstein, J. L., and Minkowski, R. 1964, Ap. J. 140, 1601.

Harmon, R. J., and Seaton, M. J. 1964, Ap. J. 140, 824.

Limber, D. N. 1960, Ap. J. 131, 168.

Mathews, W. G. 1966, Ap. J. 143, 173.

Menzel, D. 1946, Physica, 12, 168.

Merrill, P. W. 1958, in "Etoiles à raies d'émission" (Mém. Soc. Roy. Sci. Liège, 4th Ser. 20), p. 436.

Minkowski, R., and Abell, G. O. 1963, Pub. A. S. P. 75, 488.

O’Dell, C. R. 1962, Ap. J. 135, 371.

-- 1963, Ap. J. 138, 67.

Osterbrock, D. E. 1964, Annual Rev. Astronomy and Astrophysics 2, 95.

Schmidt, M. 1963, Ap. J. 137, 758.

Swarzschild, M. 1958, Structure and Evolution of the Stars (Princeton: Princeton University Press), pp. 254-259.

Shklovski1, I. S. 1956, Astr. Zhurnal, U.S.S.R. 33, 315.

Wilson, O. C. 1950, Ap.J. 111, 279. 